Probing dielectric ceramics surface at sub-micrometer scale

This content has been downloaded from IOPscience. Please scroll down to see the full text. 2010 IOP Conf. Ser.: Mater. Sci. Eng. 8012038

(http://iopscience.iop.org/1757-899X/8/1/012038)

View the table of contents for this issue, or go to the journal homepage for more

Download details:

IP Address: 147.96.14.15

This content was downloaded on 23/07/2015 at 08:27

Please note that terms and conditions apply. 


\title{
Probing dielectric ceramics surface at sub-micrometer scale
}

\author{
Patrick Fiorenza ${ }^{1}$, Raffaella Lo Nigro, Vito Raineri \\ Istituto per la Microelettronica e Microsistemi, Consiglio Nazionale delle Ricerche; \\ Stradale Primosole 50, 95121 Catania (Italy) \\ Rainer Schmidt and Derek C Sinclair \\ Department of Engineering Materials, Sir Robert Hadfield Building, University of \\ Sheffield; Mappin Street, Sheffield, UK, S1 3JD (United Kingdom) \\ E-mail: patrick.fiorenza@imm.cnr.it
}

\begin{abstract}
Scanning probe microscopy (SPM) with conductive tips has been used to image the dielectric properties of ceramics with giant permittivity. In particular, measurements in impedance mode of local resistivity allowed to image the permittivity map on polycrystalline materials. Such imaging provides correlation between the dielectric properties and the sample structure, in particular focusing on defects inside the single grains.

Great attention has been devoted to the possible artefacts due to surface imperfections, such as huge roughness and/or contamination. A reliable surface investigation has been obtained after the definition of both the physical and geometrical criteria to avoid the artefacts due to both the surface or anomalous tip-sample contact area variation (for instance, in grain boundaries, holes and cracks in the ceramic pills).

In particular, the power spectral density (PSD) allows to get access to the different periodic components of the surface roughness. The PSD demonstrated to be a sensitive tool to check the surface conditions after the polishing procedures aimed to the progressive decreasing of surface roughness, in order to reach the SPM limits and to avoid artefacts inducing wrong data interpretation.
\end{abstract}

\section{Introduction}

Often the investigation of dielectric materials require to get information on the homogeneity of the sample. To do that, the ideal case is the investigation of an "ideal sample cross-section" (figure 1). This is required because several artefacts may affect the interpretation of the SPM data. As an example the convolution between the probe and the superficial features cannot keep constant the contact area and the formation of an non-ideal electric contact has to be proved.

SPM is sensitive to the sample surface conditions and in some cases could produce artefact and/or erroneous data interpretation. In fact, surface roughness, chemical contamination, mobile and fixed charges can produce artifact and can drive to wrong data interpretation[1]. SPM technique and the sample surface preparation have to be improved in order to get powerful and reliable methods in the heterogeneous material investigation[2-5].

1 To whom any correspondence should be addressed. 


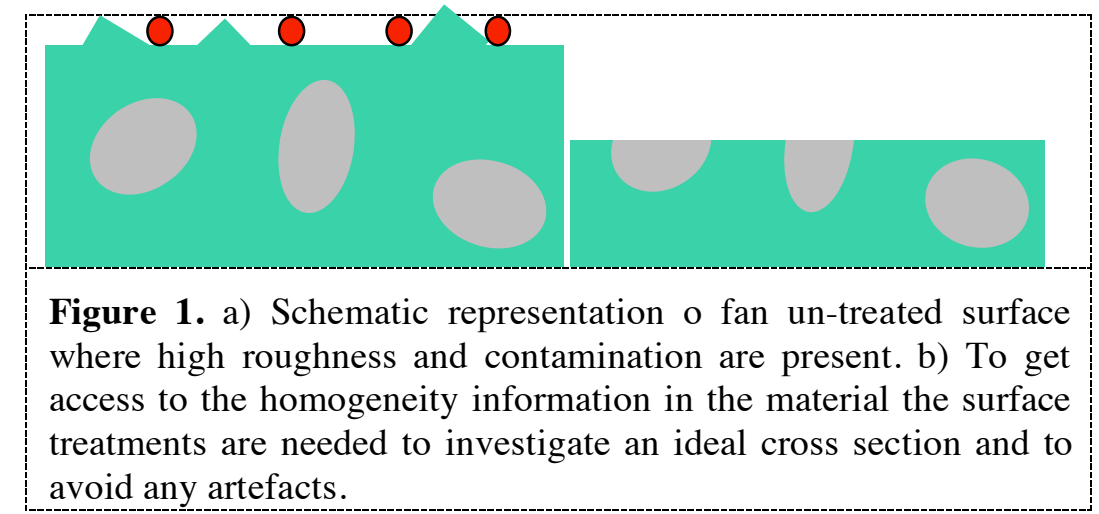

\section{Experimental part}

The investigated samples is a $\mathrm{CaCu}_{3} \mathrm{Ti}_{4} \mathrm{O}_{12}$ (CCTO) ceramic it has been fabricated by the mixed oxide route as described in detail elsewhere[6]. The ceramic pills were polished to eliminate the influence of superficial artefacts in the C-AFM mapping. Measurements were performed using a back side contact, obtained by silver paint, opposite to the polished surface. Measurements at nanometer scale were performed by a Digital Instrument D3100 atomic force microscope (AFM) with a Nanoscope V controller operating in air and in tapping mode. The quality of the electrical contact has been proved using the scanning impedance microscope (SIM)[2] Measurements were performed using a back contact, obtained by silver paint, opposite to the polished surface. SIM measurements were carried out in constant $\Delta V$ mode. The ac bias applied between the tip and sample was varied in the 1-10 V peakto-peak range at $90 \mathrm{kHz}$ with the resonator frequency $1.0 \pm 0.1 \mathrm{GHz}$.

The superficial artifacts contributions have been reduced by mechanical polishing of the CCTO pellet surfaces using decreasing diamond pastes from 30 to $1 \mu \mathrm{m}$ in diameter. The final polishing was obtained with a $1 / 20 \mu \mathrm{m}$ diamond paste on a frosted glass plate. The final roughness (RMS) value of the CCTO surface was $\sim 1 \mathrm{~nm}$.

\section{Discussion}

Superficial roughness can be defined in several different ways. Numerous books are available which describe image processing with matrices, Fourier analysis and statistical methods as autocovariance[7]. The physical properties of a surface have to explained in terms of all the component that are present on the surface. Features with different size and different distribution can be present and each of the superficial features are related to different physical structures. Fourier analysis of the topographic images clarifies which is the contribution of each component to total roughness.

In the present work, to get access to the surface geometrical deep information, the Power Spectral Density (PSD) has been considered. It is correlated to the Fourier surface analysis (FFT). PSD and Root Mean Square (RMS) are interrelated mathematically as follows:

$\mathrm{PSD}=\mathrm{FFT}^{2}=\mathrm{RMS}^{2}$

Surfaces with different properties can give the same RMS value, but PSD is helpful to know which wavelengths occur more often and which impart the greatest influence or "power" to the surface's topography. This means that the comparison of periodic features on the sample surface appear on the PSD in function of their dimension.

$$
F F T=\sum_{j=0}^{N / 2-1} e^{\frac{2 \pi i k j}{N / 2}} f_{2 j}+W^{k} \sum_{j=0}^{N / 2-1} e^{\frac{2 \pi i k j}{N / 2}}\left(f_{2 j}+1\right)
$$

Where $W \equiv e^{\frac{2 \pi i}{N}}$ and $\mathrm{k}$ varies from 0 to $\mathrm{N}$.

Figure 2 shows the atomic force microscopy (AFM) micrographs after the progressive superficial treatments in order to reach the smoothest condition possible. After the first image (figure 2a) where big features are present, one intermediate condition is reached where the features are removed and 
some "intrinsic" voids appeared on the treated surface (figure 2c). The nature of such voids is related to the spontaneous porosity of the material under investigation. After the first part, using finer treatment condition no changes have been observed concerning the voids.

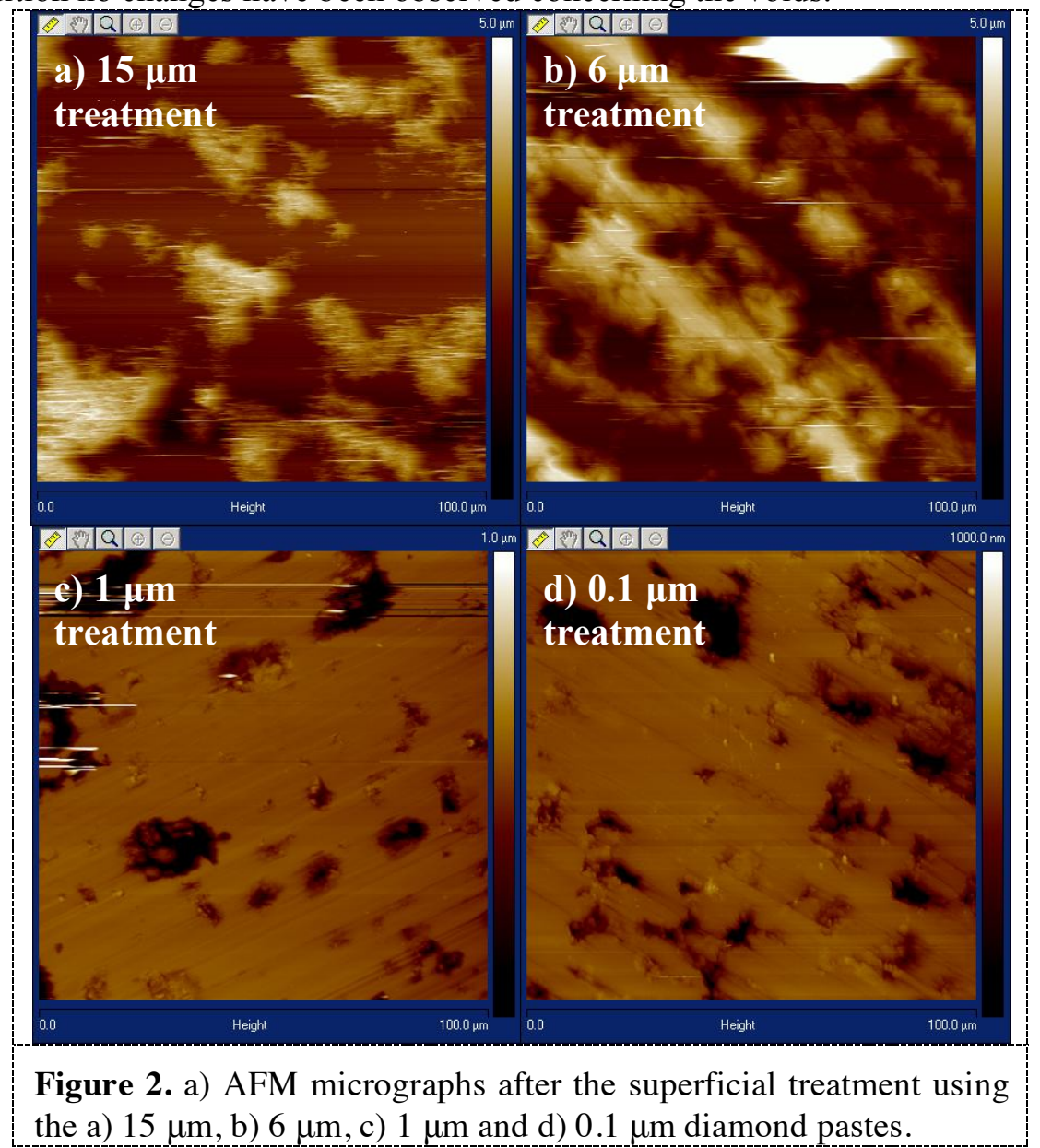

As already explained, the full understanding come out from the PSD data versus frequency (that is the inverse of the wavelength, and the wavelength is two times the greater than the considered periodic component). The first part of the surface treatment produces a strong roughness reduction (figures $2 \mathrm{a}$ and $2 b$ ) reducing down the macroscopic sample defect as hillocks and surface contamination (red circle figure 3a). In fact, looking at the low frequency PSD component (red circle in figure 3a) it is evident that the saturation at the lowest PSD value is reached already after the treatment with $1 \mu \mathrm{m}$ diamond paste. Figures $2 \mathrm{c}$ and $2 \mathrm{~d}$ demonstrate the presence of voids embedded in the material. Obviously, they cannot be reduced with superficial treatments. This explains why the PSD at low frequencies saturates early during the superficial treatments. On another hand, after the lower scale surface treatment (figures $2 \mathrm{c}$ and $2 \mathrm{~d}$ ), at high frequency there is a strong reduction to the surface contribution (green circle in figure 3a). In fact, the procedures do not chance the low frequencies contribution (already saturated) because they are dominated by intrinsic features as the voids; but they allow to reach almost the atomic flat surface for low scan size with a RMS value about $1 \mathrm{~nm}$. In fact, figure 3a shows a clear strong PSD reduction at high frequency (green circle) after the $0.1 \mu \mathrm{m}$ diamond paste treatment reaching roughness values comparable to an atomic flat sample for small (about $10 \times 10 \mu \mathrm{m}^{2}$ ) scanned regions (RMS $\sim 1 \mathrm{~nm}$ ). It has to be remarked that the mechanical procedures involved in the treatments of the sample surface do not introduce extra-features. In fact, the bidimensional PSD do not show any peak at the frequencies characteristic of the diamond pastes used as final parts of the treatment. In this way artifacts, due to the sample features-tip convolution, are avoided. To get a complete proof of the quality and reliability of the characterisation technique other 
kind of artefact must to be avoided. SPM methods are usually correlated also with the electrical properties acquirement. This requires that no artefacts has to affect the electrical characterisation. A physical criterion to evaluate the quality of the electric contact between the sample surface and the nanometre probe is presented. The criterion checks the electrical contact quality between the probe and the sample surface using the SIM in order to clarify the presence of not of electrical features as fixed or mobile charges and also the presence of surface states. In fact, not also the raw condition but also due the mechanical treatments the presence of both fixed and mobile charges or also surface states that can be introduced inducing artefacts or erroneous data interpretation.

Figure $3 \mathrm{~b}$ shows the $\mathrm{dC} / \mathrm{dV}$ versus voltage curves acquired both on the treated (blue curve) an untreated (red curve) surface. The curve before the treatment is almost constant in the whole applied voltage range, this behaviour can be explained with the presence of mobile charges on the sample surface because they compensate the charge accumulated at the metal interfaces reducing the capacitance response. At pristine condition a non-quantified amount of charges are present and the contact is degenerated. On another hand, after the treatment the surface shows an ideal contact behaviour as in the case of an ideal Metal-Insulator-Semiconductor device or an ideal Schottky contact (blue curve)[8]. The peak position is centred around $0 \mathrm{~V}$, this corresponds at a low concentration of fixed charges because if present they produce a shift of the peak.

\section{Conclusion}
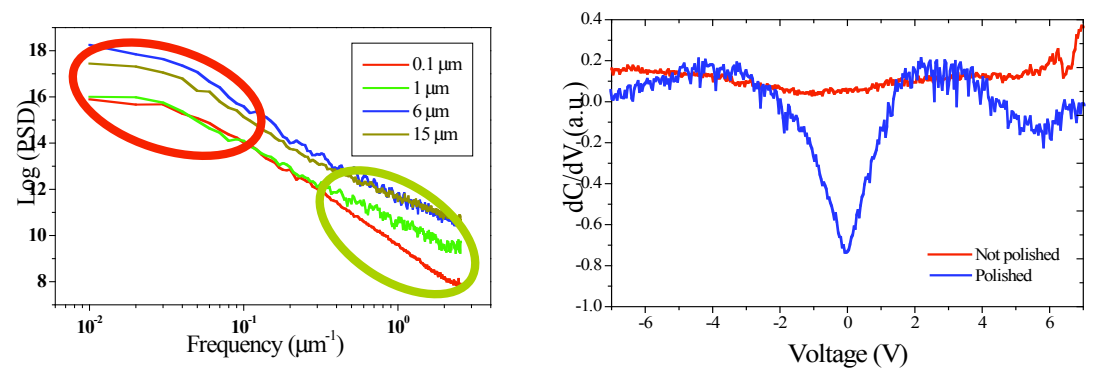

Figure 3. a) PSD versus frenquency after different supersicial tratments. b) $\mathrm{dC} / \mathrm{dV}$ versus voltage curves on both treated and untreated surface.

SPM requires to be reliable and reproducible characterisation methodology for dielectric materials investigation. The present work presented an experimental procedure to treat the sample surface in order to avoid the presence of any artefact. In particular two criteria have been proposed to control carefully the influence of the superficial features on the roughness (monitoring the PSD data during the superficial treatments) and in order to provide an ideal electric contact between the probe and the sample surface (checking the $\mathrm{dC} / \mathrm{dV}$ curves behaviour).

\section{References}

[1] Raineri V, Fiorenza P, Lo Nigro R, Sinclair D C; 2007 XII GADEST proceedings series

[2] Fiorenza P, Lo Nigro R, Bongiorno C, Raineri V, Ferarrelli M C, Sinclair D C, West A R; 2008 Appl. Phys. Lett. 92, 182907

[3] Fiorenza P, Lo Nigro R, Raineri V, Toro R G, Catalano M R; 2007 J. Appl. Phys. 102, 116103

[4] Fiorenza P, Lo Nigro R, Sciuto S, Delugas P, Raineri V, Toro R G, Catalano M R, Malandrino G; 2009 J. Appl. Phys. 105, 061634

[5] Fiorenza P, Raineri; 2006 Appl. Phys. Lett. 88, 212112

[6] Sinclair D C, Adams T B, Morrison F D, West A R; 2002 Appl. Phys. Lett. 80, 2153

[7] Russ J C; 1992 The Image Processing Handbook; CRC Press, Inc., Boca Raton

[8] Fiorenza P, Lo Nigro R, Raineri V, Lombardo S, Toro R G, Malandrino G,. Fragalà I L; 2005 J. Appl. Phys. 98, 044312 